\title{
Lyapunov Modes and Time-Correlation Functions for Two-Dimensional Systems
}

\author{
Tooru Taniguchi and Gary P. Morriss \\ School of Physics, University of New South Wales, Sydney, New South Wales \\ 2052, Australia
}

\begin{abstract}
The relation between the Lyapunov modes (delocalized Lyapunov vectors) and the momentum autocorrelation function is discussed in two-dimensional hard-disk systems. We show numerical evidence that the smallest time-oscillating period of the Lyapunov modes is twice as long as the time-oscillating period of momentum autocorrelation function for both square and rectangular two-dimensional systems with hard-wall boundary conditions.
\end{abstract}

Key words: Lyapunov mode, velocity autocorrelation function, Lyapunov vector, time-oscillating period, many-particle system

\section{Introduction}

The Lyapunov mode is a delocalized structure appearing in the Lyapunov vectors corresponding to the Lyapunov exponents close to zero in many-body chaotic systems. The existence of such a mode structure for Lyapunov vectors was first suggested from the stepwise structure of the Lyapunov spectra for many-hard-core-particle systems $[1,2]$, and led to the discovery of a stationary transverse T-mode structure in the Lyapunov vectors $[2,3,4]$. Further investigations clarified the other two kinds of Lyapunov modes, the longitudinal L-mode [5] and the momentum proportional P-mode $[6,7,8]$, both of which contain an explicit time-dependence. Combining these three kinds of Lyapunov modes, explained the degeneracies in the stepwise structure of Lyapunov spectra $[7,8,9]$. Moreover, the structure of these Lyapunov modes suggests that the origin of the Lyapunov modes is the dynamical conservation laws, like energy and momentum conservation, and spatial and temporal translational invariances $[6,7,8,9]$ and Noether's theorem. The conservation laws and translational invariances dominate the global and thermodynamic behavior of many-body 
systems, and the behavior of Lyapunov vectors for the Lyapunov exponents close to zero should correspond to slow and collective movements. Therefore it is expected that the Lyapunov modes (which are chaotic properties) allow us to discuss the thermodynamic behavior of many-body systems dynamically. Many studies of the Lyapunov modes have used hard-core-particles in quasi-one-, two- or three-dimensional systems because of its established Lyapunov vector dynamics and clear mode structures. Recently Lyapunov modes for soft-core particle systems have also been investigated [10,11,12]. Some analytical approaches have been proposed to understand the Lyapunov modes and the stepwise structure of Lyapunov spectra. These include a random matrix approach [13], a Fokker-Planck equation approach [14], kinetic approaches $[15,16,17]$ and a periodic orbit approach [18].

One recent development in the study of Lyapunov modes is the discovery of a connection between the time dependence of the Lyapunov modes and the momentum autocorrelation function $[7,8]$. The integral of the momentum autocorrelation function gives the transport coefficient (here the diffusion coefficient) through linear response theory [19]. Moreover it includes information on the collective movement of many-body systems [20,21,22], and is accessible experimentally using neutron and light scattering techniques [23,24,25]. It was shown in Ref. $[7,8]$ that the largest time-oscillating period $T_{L y a}$ of timedependent Lyapunov modes is twice as long as the time-oscillating period $T_{t c f}$ of the momentum autocorrelation function, namely

$$
T_{L y a}=2 T_{t c f} .
$$

This relation connects the Lyapunov mode and an experimentally accessible quantity. In reference $[7,8]$, a theoretical argument to support this relation, and numerical evidence that this relation is independent of density, particle number, and boundary conditions in quasi-one-dimensional systems was given. The aim of this paper is to present numerical evidence that the relation (1) is satisfied not only in quasi-one-dimensional systems but also in fully twodimensional systems.

To calculate Lyapunov exponents and Lyapunov vectors we use the Lyapunov vector dynamics for hard-disk systems [1], and the algorithm developed by Benettin et al. [26], and Shimada and Nagashima [27]. In general, the difference between two nearby phase space trajectories defines a direction in phase space. The rate of growth of this difference is controlled by the largest Lyapunov exponent, and the normalized direction (which is time dependent) is the Lyapunov vector for that exponent. Eliminating the direction defined by the Lyapunov vector for the largest exponent, reduces the dimension of the phase space by one. In that reduced phase space we can again define a direction that corresponds to the (next) largest Lyapunov exponent and its associated Lyapunov vector. Continuing this procedure we define the same 


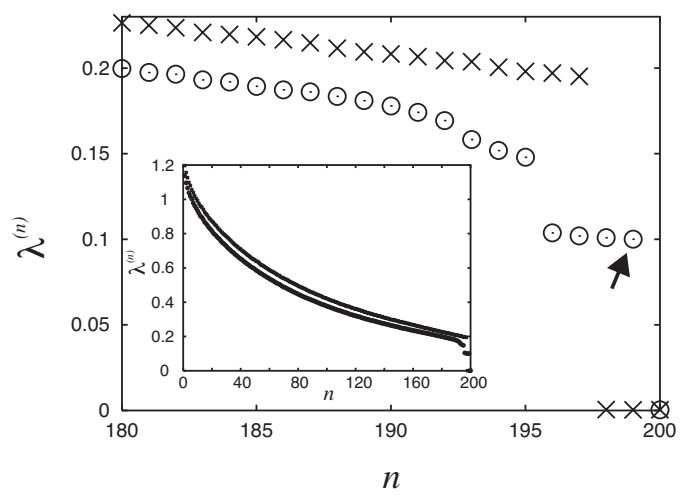

Fig. 1. The stepwise structure of Lyapunov spectrum for a square system consisting of 100 hard-disks with hard-wall boundary conditions (circles). For a comparison, the Lyapunov spectrum for a square system with periodic boundary conditions (crosses) is also shown. The Lyapunov exponent indicated by an arrow is the one in which a time-oscillation of Lyapunov mode is shown in Fig. 2(b). Inset: Full positive branch of Lyapunov spectra.

number of Lyapunov exponents and Lyapunov vectors as there are independent directions in phase space. This process is essentially the Benettin scheme [26] for calculating the full spectrum of Lyapunov exponents for the system. The only additional element is the significance given to basis vectors used in the Benettin scheme; here they called Lyapunov vectors and become an object of study in their own right. While the Lyapunov vectors associated with the largest exponents are strongly localized (having non-zero components for only a few particles) the vectors associated with the smallest positive and negative exponents have delocalized Lyapunov vectors that we refer to as Lyapunov modes.

\section{A Square System with 100 Disks}

We investigate the time-oscillating periods of the Lyapunov modes and the momentum autocorrelation function, for a system of hard-disks in a square with the hard-wall boundary conditions. Figure 1 is the Lyapunov spectrum for this system (circles). Here, we used as system parameters: $N=100$, disk radius $R=1$, mass $M=1$, total energy $E=N$, and the system lengths in the $x$ and $y$ directions $L_{x}=L_{y} \approx 38.7$, so that the particle number density is $N(2 R)^{2} /\left(L_{x} L_{y}\right) \approx 0.267$. The Lyapunov spectrum shows a 4 -point step close to the two zero-Lyapunov exponents. We use hard-wall boundary conditions because that leads to more and clearer steps in the Lyapunov spectrum than for the equivalent system with periodic boundary conditions. In fact we do not observe stepwise structure of the Lyapunov spectrum for a 100-disk system 
(a)

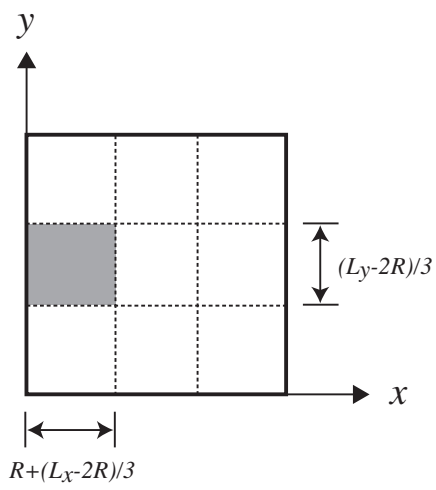

(b)

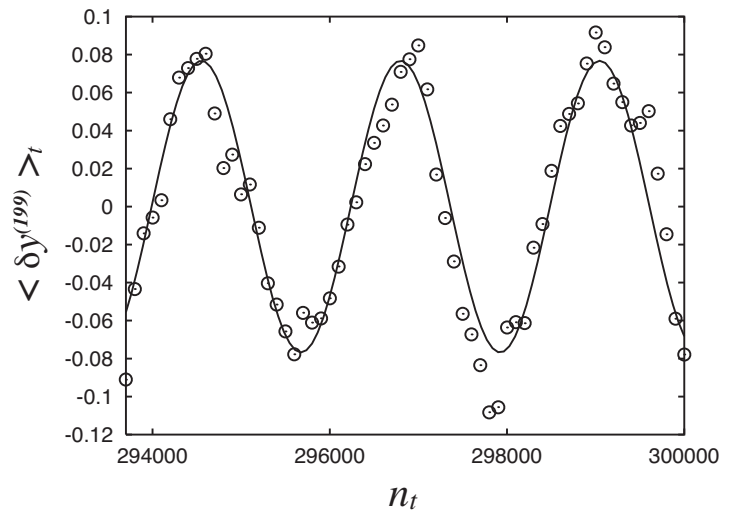

Fig. 2. (a) The spatial region of the system in which the average of the longitudinal Lyapunov mode of Fig. (b), is taken. (b) The time-oscillation of the local spatial region for the Lyapunov vector component $\left\langle\delta y^{(2 N-1)}\right\rangle_{t}$ for the component $d y_{j}^{(2 N-1)}$ of the Lyapunov vector. The line is a fit of the numerical data to a sinusoidal function. The system is the same as that in Fig. 1 with hard-wall boundary conditions.

with periodic boundary conditions. To show this, in Fig. 1, we also plotted the Lyapunov spectrum for a square system with periodic boundary conditions (crosses). (Here we used the same system parameters as for hard-wall boundary conditions, except that $L_{x}=L_{y} \approx 36.7$ so the effective region available for the disks to move is the same in both cases.) The system with periodic boundary conditions is too small to observe the stepwise structure of the Lyapunov spectrum. The number of zero-Lyapunov exponents is 2 for hard-wall boundary conditions, because of energy conservation and time-translational invariance (determinisity of orbit), and is 6 for periodic boundary conditions, because of total momentum conservation, conservation of center of mass in both directions, energy conservation and time translational invariance [28]. Only half of those zero-Lyapunov exponents are presented in Fig. 1.

We are interested in the time-oscillation of modes corresponding to the stepwise structure of the Lyapunov spectrum shown in Fig. 1. It is known that there are two kinds of time-dependent Lyapunov modes, the L-modes and the P-modes, each with the same period $[6,7,8,9]$. Our concern is to determine the time-oscillating period of Lyapunov mode, so it is enough to consider one of these. We choose the L-mode and concentrate into its time-oscillating behavior only, ignoring the spatial structure $[7,8,9]$. To determine the period of the L-mode we use the same system with hard-wall boundary conditions, see Fig. 1, and consider the $\delta y_{j}^{(2 N-1)}$. This Lyapunov vector component $\delta y_{j}^{(2 N-1)}$ corresponds to the Lyapunov exponent $\lambda^{(2 N-1)}$ and is indicated by the arrow in Fig. 1. To get clear time-oscillating behavior we take the average of $\delta y_{j}^{(2 N-1)}$ for disks whose centers $\left(x_{j}, y_{j}\right)$ are in the region $R<x_{j}<R+\left(L_{x}-2 R\right) / 3$ 
(a)

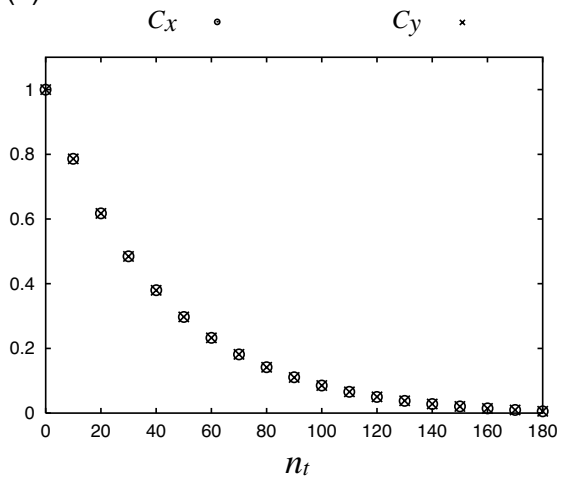

(b)

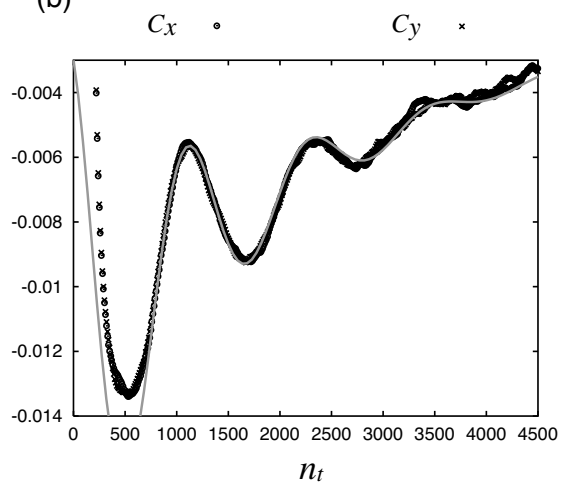

Fig. 3. The momentum autocorrelation functions as a function of the collision number $n_{t}$ for a square system consisting of 100 disks. (a) The initial damping behavior of the momentum autocorrelation functions. (b) The time-oscillating region of the momentum autocorrelation functions. $C_{x}$ (circles) is the autocorrelation function of the $x$-component and $C_{y}$ (crosses) is the autocorrelation function of the $y$-component. These two components should be equal for a square system. The system is the same as that in Fig. 2. The line in Fig. (b) is a fit of $C_{x}$ to an exponentially damped sinusoidal function.

and $R+\left(L_{y}-2 R\right) / 3<y_{j}<R+2\left(L_{y}-2 R\right) / 3$ (the grey region in Fig. $2($ a) $)$ at the time of the collisions. This region is chosen because the L-mode has a node at this end of the system, so that the longest L-mode for $\delta y_{j}^{(2 N-1)}$ should have nodes at $y=R$ and $L_{y}-2 R$ and an anti-node at $y=L_{y} / 2$, so the Lyapunov mode $\delta y_{j}^{(2 N-1)}$ should have largest amplitude. Further, we take a local-time average of the spatially averaged Lyapunov vector component $\delta y_{j}^{(2 N-1)}$ over $N$ successive disk-disk collisions. This kind of local spatial-temporal average is required to clearly observe the time-oscillation of Lyapunov modes. A square system of $N=100$ particles is not large enough to observe clear mode structures in the Lyapunov vectors, as there are only about $\sqrt{N}=10$ particles in each coordinate direction. Fig. 2 is a graph of the local spatial-temporal average $\left\langle\delta y^{(2 N-1)}\right\rangle_{t}$ of $d y_{j}^{(2 N-1)}$ as a function of the collision number $n_{t}$. In this figure we can observe the time-oscillation of the longitudinal Lyapunov mode, and the fit of the graph to a sinusoidal function $\left\langle\delta y^{(2 N-1)}\right\rangle_{t}=\alpha \sin \left(2 \pi n_{t} / T_{L y a}+\beta\right)$ with the fitting parameters $\alpha, \beta$ and $T_{L y a}$. This gives its time-oscillating period $T_{\text {Lya }}$ as $T_{\text {Lya }} \approx 2250$. Note that $T_{\text {Lya }}$ is in units of collision numbers $n_{t}$, and the real time interval of the time-oscillating period of this Lyapunov mode is given by $\tau T_{L y a}$ where $\tau \approx 0.0279$ is the mean free time.

Next, we consider the time-oscillating behavior of the momentum autocorrelation function. For this purpose we introduce the autocorrelation functions $C_{\eta}(t)$ of the components of momentum (where $\eta=x$ or $y$ ) and use the normalized expression $C_{\eta}(t) \equiv \tilde{C}_{\eta}(t) / \tilde{C}_{\eta}(0)$, in which $\tilde{C}_{\eta}(t)$ is defined 

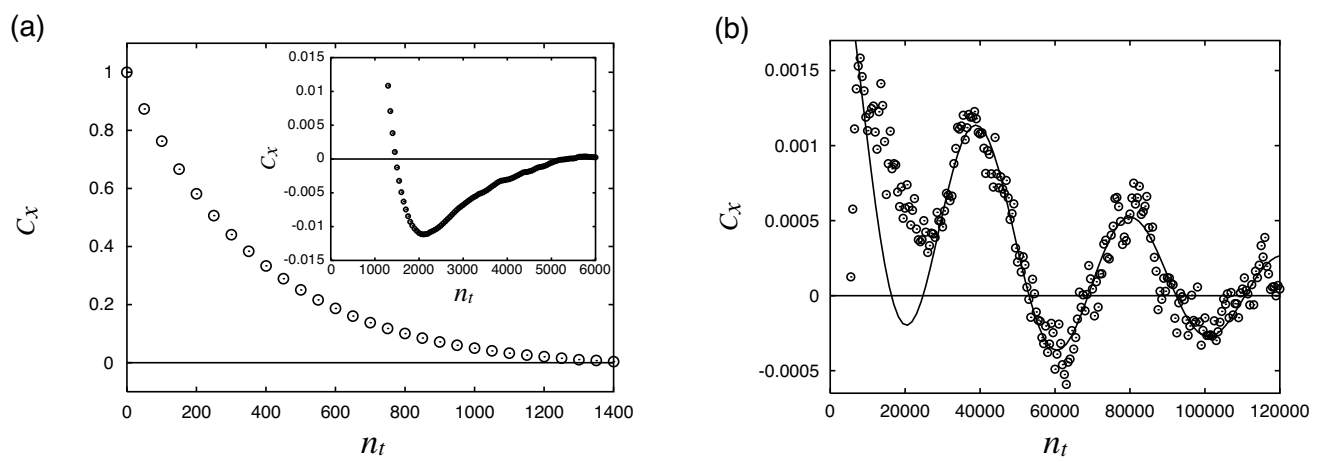

Fig. 4. The momentum autocorrelation function $C_{x}$ as a function of the collision number $n_{t}$ in a rectangular system consisting of 780 disks. (a) The initial damping of $C_{x}$ for $0<n_{t}<1400$. Inset: The negative region of $C_{x}$ for $1500<n_{t}<6000$. (b) The time-oscillating part of $C_{x}$ for $10000<n_{t}<120000$. The line is a fit of $C_{x}$ to an exponentially decaying sinusoidal function.

by $\tilde{C}_{\eta}(t) \equiv \lim _{T \rightarrow+\infty} \frac{1}{N T} \sum_{j=1}^{N} \int_{0}^{T} d s p_{\eta j}(s+t) p_{\eta j}(s)$. Here $p_{\eta j}(s)$ is the $\eta$ component of the momentum of the $j$-th disk. (In numerical calculations, the time-average for the time $s$ in the above definition of autocorrelation function is replaced by the arithmetic average of $p_{\eta j}(s+t) p_{\eta j}(s)$ after each collision.) Figure 3 shows the autocorrelation functions $C_{x}$ and $C_{y}$ for the $x$ - and $y$-components of momenta, respectively, as functions of the collision number $n_{t}$ in the same system as that used in Fig. 2. The autocorrelation functions $C_{x}$ and $C_{y}$ coincide as they should for a square system. Fig. 3(a) shows the initial damping behavior of the autocorrelation functions, and Fig. $3(\mathrm{~b})$ is the negative region in which the time-oscillating behavior of the autocorrelation functions appears. We can extract the time-oscillating period $T_{t c f}$ by fitting this region of the autocorrelation function to the function $C_{x}=\alpha \exp \left\{-\beta n_{t}\right\}+\gamma \exp \left\{-\epsilon n_{t}\right\} \sin \left(2 \pi n_{t} / T_{t c f}+\zeta\right)$ (the line in Fig. 3(b)) with the fitting parameters $\alpha, \beta, \gamma, \epsilon$ and $\zeta$. This fit gives the value of the period for the momentum autocorrelation function as $T_{t c f} \approx 1190$. Comparison of the value $T_{t c f}$ with the longest time-oscillating period $T_{L y a}$ for the Lyapunov modes gives further evidence to support the relation (1) in two-dimensional systems.

\section{A Rectangular System with 780 Disks}

A system of 100 particles is not sufficient to investigate the Lyapunov modes in two-dimensions as only a few steps of Lyapunov spectrum are observed, even for hard-wall boundary conditions. A larger two-dimensional system of 780 particles with hard-wall boundary conditions has already been investigated in 
Ref. [9] and the Lyapunov modes calculated. The system parameters used were: particle mass $M=1$, disk radius $R=1 / 2$, aspect ratio $L_{y} / L_{x}=$ 0.867 , and particle number density $N(2 R)^{2} /\left(L_{x} L_{y}\right)=0.8$. In this system the observed time-oscillating period for the Lyapunov mode is $T_{L y a} \tau \approx 12.80$, namely $T_{\text {Lya }} \approx 74000$ collisions numbers with the mean free time of $\tau \approx$ 0.000173 .

Figure 4 is the normalized autocorrelation function $C_{x}$ of the $x$-component of the momentum as a function of the collision number $n_{t}$. Here, the system shape is rectangular and $L_{x}>L_{y}$. This means that the time-oscillating period of $C_{x}$ is larger than that for $C_{y}$, and should correspond to the $T_{L y a}$ observed in Ref. [9]. The autocorrelation function $C_{x}$, normalized by its initial value, shows an initial exponential damping, as shown in Fig. 4(a), and after that a negative region appears, as in Fig. 3 for the $N=100$ case. The timeoscillating behavior of $C_{x}$ appears after the negative region of $C_{x}$, as shown in Fig. 4(b). To extract the time-oscillating period $T_{t c f}$ for $C_{x}$ we fitted this time-oscillating region of the autocorrelation function to the function $C_{x}=$ $\alpha \exp \left\{-\beta n_{t}\right\}+\gamma \exp \left\{-\epsilon n_{t}\right\} \sin \left(2 \pi n_{t} / T_{t c f}+\zeta\right)$ with the fitting parameters $\alpha, \beta$, $\gamma, \epsilon$ and $\zeta$. This fit gives us a value of $T_{t c f} \approx 40800$ for time-oscillating period of $C_{x}$. This result again confirms that the relation (1) is satisfied approximately for two-dimensional systems.

We emphasize that this time-oscillating behavior of the autocorrelation function $C_{x}$ appears much later than its initial damping, and with a very small amplitude. (The above fit gives $\gamma \approx 0.00127$ as the amplitude of the timeoscillation of $C_{x}$ shown in Fig. 4(b), which is much smaller than the initial value 1 for $C_{x}$.) Besides, the time-oscillation of the autocorrelation function has the time-scale much longer than its decay time. This means that very long numerical calculations are required to obtain the time-oscillating behavior of $C_{x}$ in such a large system, and we calculated more than $5 \times 10^{7}$ collisions to get the data presented in Figs. 3(b) and 4(b). These are much longer than the simulations required to calculate Lyapunov spectra.

\section{Conclusion}

In conclusion, this paper gives further numerical evidence that the relation $T_{\text {Lya }} \approx 2 T_{t c f}$ for the smallest time-oscillating period $T_{\text {Lya }}$ of the Lyapunov mode and the time-oscillating period $T_{t c f}$ of the momentum autocorrelation function is satisfied in fully two-dimensional hard-disk systems. This supports our previous results reported in Ref. [7,8], adding numerical evidence that the relation is independent of the spatial dimension of the system.

As a remark, in this paper we used a simple fit of time-oscillation of the 
autocorrelation function and Lyapunov modes to functions including a sinusoidal function of time in order to extract their time-oscillating periods, as was done for the quasi-one-dimensional systems $[7,8]$. However, the amplitude of the time-oscillating of momentum autocorrelation function is smaller for twodimensional systems than for quasi-one-dimensional systems. Considering the Fourier transformation of momentum autocorrelation function and Lyapunov modes may be more efficient technique to extracting the periods.

The authors of this paper appreciate the financial support of the Japan Society for the Promotion of Science.

\section{References}

[1] Ch. Dellago, H. A. Posch, and W. G. Hoover, Phys. Rev. E 53, 1485 (1996).

[2] Lj. Milanović, H. A. Posch, and Wm. G. Hoover, Mol. Phys. 95, 281 (1998).

[3] H. A. Posch and R. Hirschl, in Hard ball systems and the Lorentz gas, edited by D. Szász (Springer-Verlag, Berlin, 2000), p. 279.

[4] Lj. Milanović and H. A. Posch, J. Molec. Liquids, 96-97, 221 (2002).

[5] C. Forster, R. Hirschl, H. A. Posch, and W. G. Hoover, Physica D 187, 294 (2004).

[6] T. Taniguchi and G. P. Morriss, Phys. Rev. E 68, 026218 (2003).

[7] T. Taniguchi and G. P. Morriss Phys. Rev. E 71016218 (2005).

[8] T. Taniguchi and G. P. Morriss, Phys. Rev. Lett. 94, 154101 (2005).

[9] J. -P. Eckmann, C. Forster, H. A. Posch, and E. Zabey, J. Stat. Phys. 118, 813 (2005).

[10] Wm. G. Hoover, H. A. Posch, C. Forster, C. Dellago, and M. Zhou, J. Stat. Phys., 109, 765 (2002).

[11] C. Forster and H. A. Posch, New J. Phys. 7, 32 (2005).

[12] H. Yang and G. Radons, Phys. Rev. E 71, 036211 (2005).

[13] J. -P. Eckmann and O. Gat, J. Stat. Phys. 98, 775 (2000).

[14] T. Taniguchi and G. P. Morriss, Phys. Rev. E 65, 056202 (2002).

[15] S. McNamara and M. Mareschal, Phys. Rev. E 64, 051103 (2001).

[16] M. Mareschal and S. McNamara, Physica D 187, 311 (2004).

[17] A. S. de Wijn and H. van Beijeren, Phys. Rev. E 70, 016207 (2004). 
[18] T. Taniguchi, C. P. Dettmann, and G. P. Morriss, J. Stat. Phys. 109, 747 (2002).

[19] R. Kubo, M. Toda, and N. Hashitsume, Statistical physics II, nonequilibrium statistical mechanics (Springer-Verlag, Berlin, 1985).

[20] R. Zwanzig, Phys. Rev. 156, 190 (1967).

[21] A. Rahman, Phys. Rev. Lett. 19, 420 (1967); W. E. Alley, B. J. Alder, and S. Yip, Phys. Rev. A 27, 3174 (1983).

[22] J. P. Hansen and I. R. McDonald, Theory of simple liquids, 2nd ed. (Academic press, London, 1990).

[23] J. R. D. Copley and S. W. Lovesey, Rep. Prog. Phys. 38, 461 (1975).

[24] A. A. van Well, P. Verkerk, L. A. de Graaf, J.-B. Suck, and J. R. D. Copley, Phys. Rev. A 31, 3391 (1985).

[25] W. Schaertl and C. Roos, Phys. Rev. E 60, 2020 (1999).

[26] G. Benettin, L. Galgani, A. Giorgilli, and J. -M. Strelcyn, Meccanica 15, 9 (1980); Meccanica 15, 21 (1980).

[27] I. Shimada and T. Nagashima, Prog. Theor. Phys. 61, 1605 (1979).

[28] P. Gaspard, Chaos, Scattering and Statistical Mechanics (Cambridge University Press, Cambridge, England, 1998). 